Joanna Morawska ${ }^{1}$

Ewa Niebudek-Bogusz ${ }^{1}$

Justyna Wiktorowicz $z^{2}$

Mariola Śliwińska-Kowalska ${ }^{1}$

\title{
SCREENING VALUE OF V-RQOL IN THE EVALUATION OF OCCUPATIONAL VOICE DISORDERS
}

\author{
${ }^{1}$ Nofer Institute of Occupational Medicine, Łódź, Poland \\ Audiology and Phoniatrics Clinic \\ ${ }^{2}$ University of Lodz, Łódź, Poland \\ Department of Economic and Social Statistics
}

\begin{abstract}
Background: Given the growing number of occupational voice users, easy and quick broad-scale screening is necessary to provide prophylaxis of voice disorders. The aim of the study was to assess applicability of the Voice Related Quality of Life questionnaire (V-RQOL) to screening occupational voice disorders. Material and Methods: The research comprised 284 subjects divided into 3 groups: 0 - the control group of normophonic subjects, non-professional voice users $(\mathrm{N}=60), 1$ - occupational voice users with objectively confirmed voice disorders $(\mathrm{N}=124), 2$ - the non-randomized group of occupational voice users with and without voice problems $(\mathrm{N}=100)$. Self-assessment of voice was performed by means of the V-RQOL in comparison to the Voice Handicap Index (VHI). The relation between the V-RQOL and VHI was determined by means of linear regression. Receiver Operating Characteristic (ROC) curves were constructed and the cut-off point of the V-RQOL was determined to discriminate between normophonic and dysphonic subjects. Results: The relationship between the VHI and V-RQOL scores indicated a satisfactory coefficient of determination: $\mathrm{R}^{2}=0.7266$. High values of Cronbach's a confirmed high reliability of the V-RQOL test (0.867). Voice-Related Quality of Life questionnaire (V-RQOL) results were significantly worse in the study group than for normophonic controls $(\mathrm{p}<0.001)$. The cut-off point for the test was set at 79 points. The determined area under the curve $($ AUC $)=0.910$ $(\mathrm{p}<0.001)$ showed high diagnostic accuracy of the V-RQOL. Results of the V-RQOL differed for diagnose-based subgroups of dysphonic patients. Conclusions: The study gives grounds for application of the V-RQOL as a reliable tool for screening occupational voice disorders. Med Pr 2018;69(2):119-128
\end{abstract}

Key words: screening, self-assessment, dysphonia, occupational voice, voice disorders, biopsychosocial impact

Corresponding author: Joanna Morawska, Nofer Institute of Occupational Medicine, Audiology and Phoniatrics Clinic, św. Teresy 8, 91-348 Łódź, Poland, e-mail: joannamorawska@poczta.onet.pl

Received: May 23, 2017, accepted: September 28, 2017

\section{INTRODUCTION}

Occupational voice disorders (occupational dysphonia) have been receiving increased attention over the past few decades. Their diagnosis still presents numerous challenges to both clinicians and researchers [1-3]. Occupational dysphonia is complex in nature and requires multidimensional management [4-6]. Given that voice is a key tool for professional voice users, its disorders may result in significant communication handicap for those affected $[1,7]$. Voice disorders for those who rely on their voice for work, in addition to causing typical problems such as social isolation, depression and impaired quality of life may also pose a threat in the form of absenteeism from work and may be employment threatening [7-10]. Taking into consideration the fact that the number of occupational voice users is constantly growing, these groups should receive most comprehensive care and broad-scale screening should be applied in order to prevent occupational voice disorders as well as to treat them as soon as the first symptoms manifest themselves.

According to the guidelines set by the World Health Organization, the ultimate goal of health care is to maintain and improve the quality of life of people, and therefore a holistic approach to the patient should be

Funding: This study was partially supported by the project of the Ministry of Science and Higher Education (No. 18.15/2016-2017, titled "Comparison of voice-related quality of life assessment conducted by means of V-RQOL questionnaire and Voice handicap Index (VHI) in dysphonic occupational voice users," project manager: Joanna Morawska, M.A.). 
adopted and the diagnosis should be of multidimensional nature [11]. Complex voice disorder assessment protocols include perceptual, laryngovideostroboscopic, acoustic and aerodynamic assessments which help in determining the nature of voice disorders. These however, are not sufficient to acquire the functional, social and emotional consequences of a vocal deviation and do not take account of how the problem affects the patient's daily life $[12,13]$. Therefore, assessment of the impact of a voice disorder on a general quality of life should be an essential part of the examination $[14,15]$.

In the last decades, a number of patient-report questionnaires have been developed to evaluate patients' subjective complaints related to voice $[12,16,17]$. The most commonly used self-report questionnaires are the Voice Handicap Index (VHI), Voice Symptom Scale (VoiSS) and Voice Related Quality of Life (V-RQOL). In Polish clinical practice, among phoniatricians, otolaryngologists and speech therapists the VHI has been so far the only such a tool used for monitoring occupational voice $[18,19]$.

None of the studies as of yet, however, has examined the perceived quality of life assessed by means of the V-RQOL for subjects with occupational voice disorders confirmed by a complex examination. The aim of this study was to evaluate the applicability of the V-RQOL as a tool for screening occupational voice disorders.

\section{MATERIAL AND METHODS}

A total of 284 individuals (mean age: 39.3 years old) were allocated into the following 3 groups:

group 0 - the control group of normophonic subjects and non-professional voice users,

group 1 - occupational voice users with voice disorders confirmed by videolaryngostroboscopy (VLS) and the acoustic analysis,

group 2 - the non-randomized group of occupational voice users with and without voice problems (without a phoniatric diagnosis).

Age of the study subjects is presented in the Table 1. The control group was recruited from among 60 normophonic subjects, 51 women and 9 men (mean age: 41 years old), who had no vocal complaints, no history of laryngeal disorders and so signs of laryngeal abnormality on indirect laryngoscopy.

The study group 1 consisted of 124 occupational voice users, 108 women and 16 men (mean age: 44 years old). The majority of this group were teachers $(\mathrm{N}=90)$,
Table 1. Age of study subjects - participants of the screening occupational voice disorders

\begin{tabular}{lccc}
\hline \multirow{2}{*}{ Study group } & \multicolumn{3}{c}{$\begin{array}{c}\text { Age } \\
\text { [years] }\end{array}$} \\
\cline { 2 - 4 } & range & $\mathrm{M}$ & $\mathrm{Me}$ \\
\hline Total $(\mathrm{N}=284)$ & $19-63$ & 39.3 & 39.0 \\
Group 0 (N =60) & $19-62$ & 40.8 & 39.0 \\
Group 1 $(\mathrm{N}=124)$ & $25-63$ & 44.5 & 45.0 \\
Group 2 (N = 100) & $21-58$ & 31.9 & 29.0 \\
\hline
\end{tabular}

Group 0 - control group of normophonic subjects, group 1 - professional voice users with voice disorders, group 2 - non-randomized group of professional voice users with and without voice problems.

M - mean, Me - median.

followed by customer service workers, sales representatives and lawyers $(\mathrm{N}=34)$.

The non-randomized group 2 consisted of 100 occupational voice users, 77 women and 23 men (mean age: 31.9 years old), who were volunteers and agreed to take part in the study. The young mean age in this group results from the fact that a great number of subjects in this group $(\mathrm{N}=59)$ were young call center workers with up to 2 years of seniority, with reported excessive vocal fatigue. The presence or lack of voice disorders was not taken into account in this group.

The differences among all the examined groups in terms of gender $(\mathrm{p}=0.122)$, including those between the study and control group ( $\mathrm{p}=0.697)$, are not significant.

All participants of the study completed the V-RQOL questionnaire. The V-RQOL measure is a questionnaire originally developed by Hogikyan and Sethuraman [20] and was constructed to assess 2 dimensions of voice use, namely Social-Emotional (SE) and Physical Functioning (PF) domains. It consists of 10 items with a 5-point rating scale. For each item the patients rate the problems they are experiencing. The V-RQOL ratings are converted to a standard score $0-100$. According to this, 0 means poor voice-related quality of life, and 100 is the highest measure of quality of life $[12,17,20]$.

The VHI measure was used in this study as a reference tool, as it is a validated tool which has proven reliable in a number of studies on Polish population $[18,19]$ and has been commonly used in clinical practice.

Additionally, in the study group 1 apart from the perceptual voice assessment, otolaryngological examination complemented by VLS (by means of XION EndoSTROB DX) was performed. Vocal function and 
voice quality were also evaluated by measuring the aerodynamic parameter: MPT (maximum phonation time) and acoustic parameters: Jitter group (Jitter, relative average perturbation (RAP), pitch perturbation quotient (PPQ)), Shimmer group (Shimmer, amplitude perturbation quotient (APQ)) and noise-to-harmonics ratio (NHR) by means of DiagnoScope software (manufactured by Diagnova Technologies, Poland). The results of the above mentioned examinations were used in order to objectively confirm voice disorders in the group 1 .

For the statistical analysis of the values of the computed parameters IBM SPSS Statistics (version 22) software was used. In order to evaluate the usability of the V-RQOL scale in comparison with the most commonly used self-report questionnaire - VHI, the relation between these measures for all subjects $(\mathrm{N}=284)$ and for each group was determined individually. In the first part of the study the Pearson's correlation coefficient and scatter plots (with the coefficient of determination for the linear regression equation) were used.

In the next step Cronbach's a was measured to assess the internal consistency of the Polish version of the test. Next, a ROC curve was constructed to measure discrimination, that is to assess the test's ability to correctly classify subjects with and without voice disorders. The area under the ROC curve (AUC) classifies the level of accuracy of a diagnostic test [21]. The cut-off point of the V-RQOL was determined to discriminate between normophonic and dysphonic subjects (the results were confirmed with the classification tree).
Additionally, all 10 items of the V-RQOL questionnaire were submitted to a ROC curve analysis to determine the discriminating power of each question. A comparison of V-RQOL results was performed for the study group, control group and non-randomized group (ANOVA test). Subsequently, the results of the V-RQOL were compared in sub-groups of dysphonic subjects established on the basis of the VLS diagnosis (non-parametric Kruskal-Wallis test).

Approval for this study was granted by the Ethical Committee of the Nofer Institute of Occupational Medicine, Łódź, Poland (decision No. 12/2013).

\section{RESULTS}

\section{V-RQOL/VHI correlation}

Comparing VHI and V-RQOL scores for all the study subjects (by means of the linear regression) it is observed that the correlation points are grouped along a hypothetical regression line, which indicates substantial strength of the association: the coefficient of determination $\mathrm{R}^{2}=0.7266$ (Figure 1).

Assessing the strength of the correlation between the V-RQOL and VHI in the examined groups separately we should indicate that for the group 1 (occupational voice users with voice disorders objectively confirmed by laryngovideostroboscopy (LVSS) examination) the strength of the correlation was the highest: $\mathrm{R}^{2}=0.6729$ (Figure 2a). For the group 2 (occupational voice users with and without voice disorders) $\mathrm{R}^{2}$ is equal to 0.613

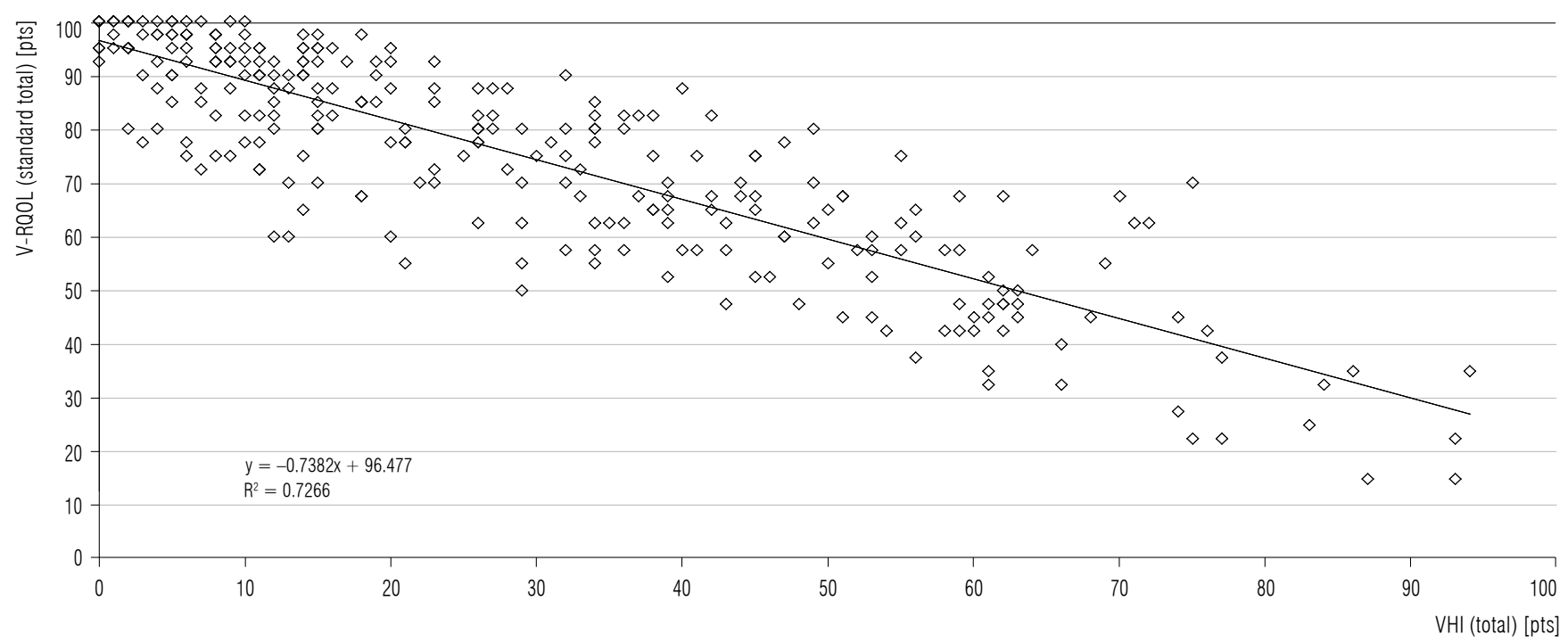

$\mathrm{R}^{2}$ - coefficient of determination.

Fig. 1. Scatter plot of the total scores from Voice Related Quality of Life (V-RQOL) and Voice Handicap Index (VHI) for the study subjects - participants of the screening occupational voice disorders 
a)

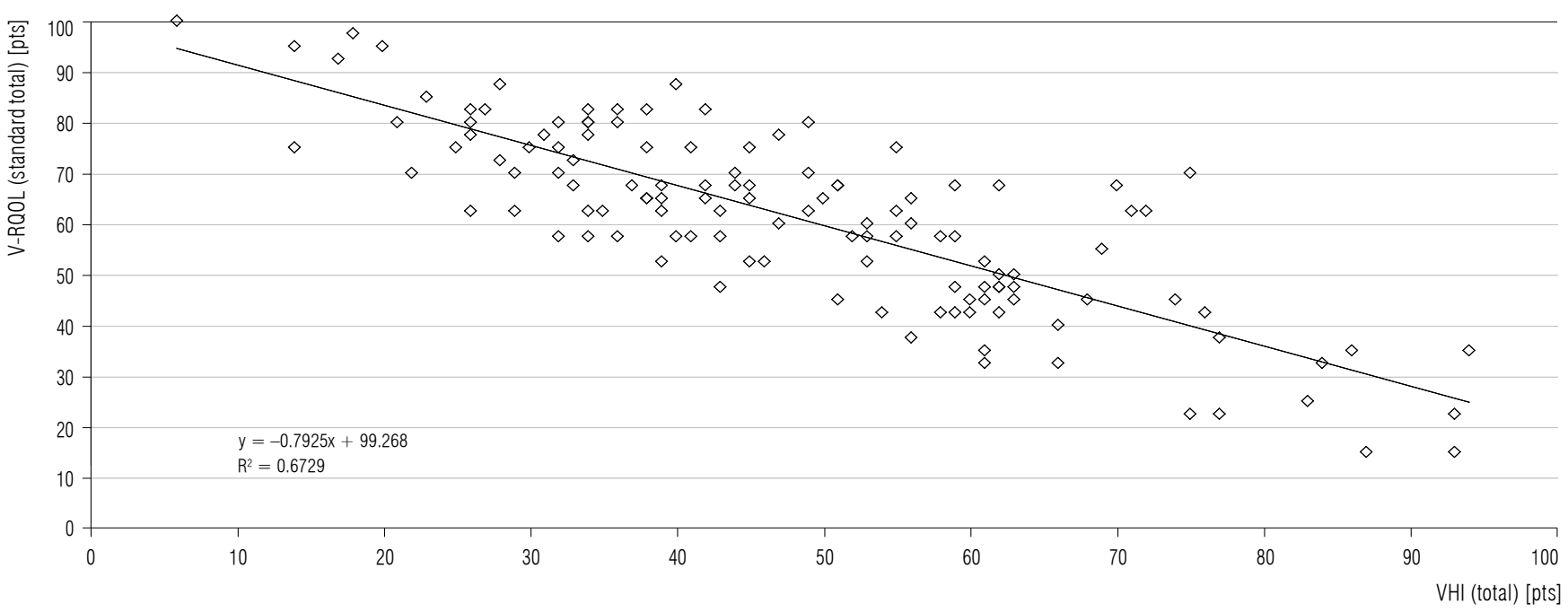

b)

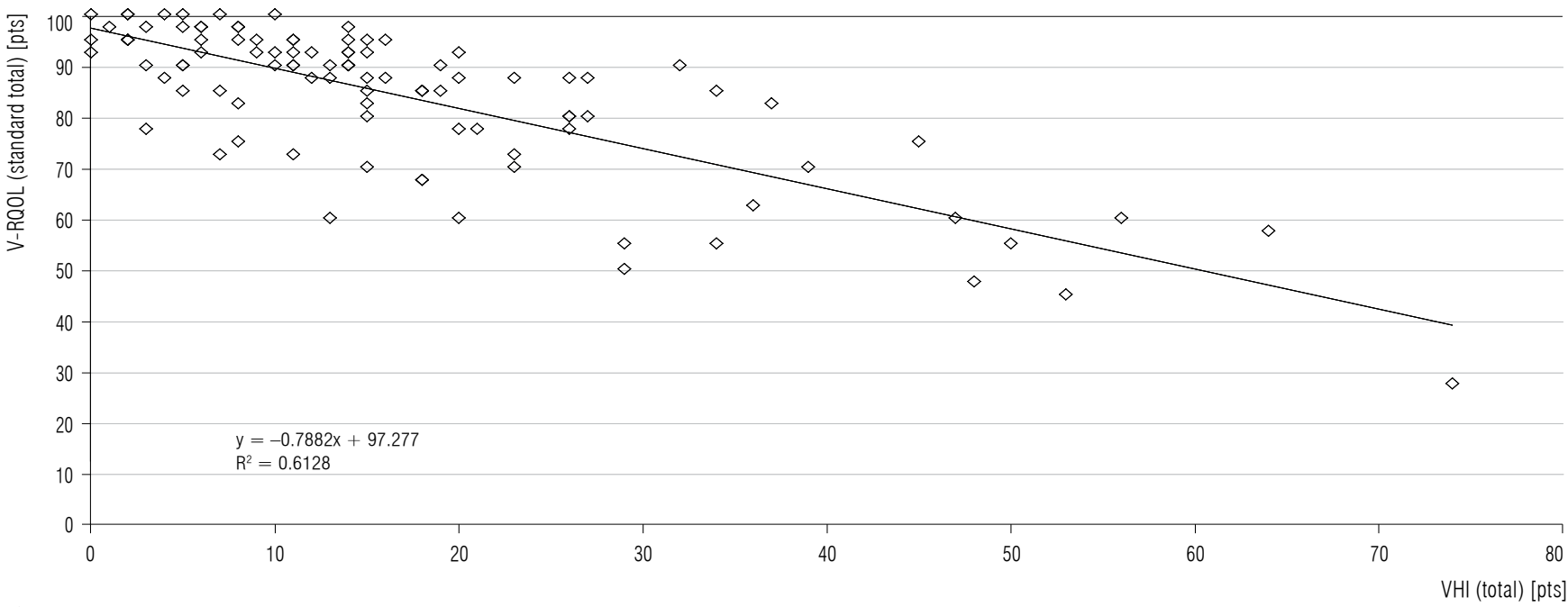

c)

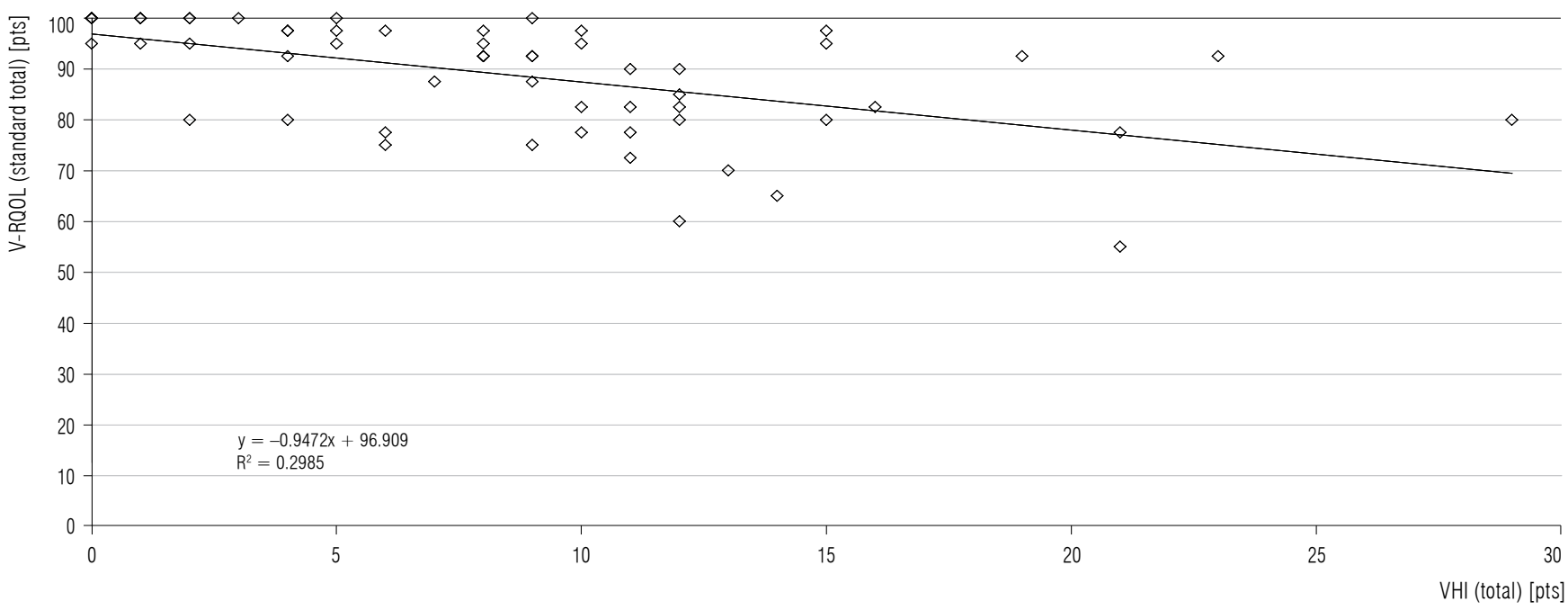

$\mathrm{R}^{2}$ - coefficient of determination.

Fig. 2. Scatter plots of the scores from Voice Related Quality of Life (V-RQOL) and Voice Handicap Index (VHI) for:

a) group 1 - professional voice users with voice disorders, b) group 2 - non-randomized group of professional voice users with and without voice problems, c) group 0 - control group of normophonic subjects 
(Figure 2b), and for group 0 (normophonic non-occupational voice users) is (as expected) much lower: $\mathrm{R}^{2}=0.298$ (Figure 2c). These results were confirmed by Pearson's correlation coefficient which amounts to -0.873 for all groups, and $-0.541,-0.821,-0.783$ for groups 0,1 and 2 , respectively $(\mathrm{p}<0.001)$. It should be noted that the correlation between the V-RQOL and VHI results is negative (the high voice handicap index corresponds with low voice-related quality of life).

Subsequently, the internal consistency reliability coefficient (Cronbach's $\alpha$ ) of the Polish version of the V-RQOL was evaluated. The study has proven that the V-RQOL is characterized by high values of Cronbach's a confirming high reliability of the test. The highest value (0.867) has been observed in the study group 1 , both in the Social-Emotional (SE) domain (0.782) and in Physical Functioning (PF) (0.808) (Table 2).

\section{Among group comparison of V-RQOL results}

Comparison of the V-RQOL scores in the study group 1 and the control group 0 indicates significantly lower total scores in the study group with mean values of 61.8 (standard deviation - SD $=17.8$ ) as compared to 89.2 $(\mathrm{SD}=11.01)$ for normophonic controls (Figure 3$)$. The differences in mean scores are also observed in Physical Functioning and Social-Emotional domains.
Table 2. Internal Consistency (Cronbach's $\alpha$ ) of the V-RQOL for studied groups - participants of the screening occupational voice disorders

\begin{tabular}{rccc}
\hline & \multicolumn{3}{c}{ Cronbach's $\alpha$} \\
\cline { 2 - 4 } Study group & total score & SE & PF \\
\hline Group 0 $(\mathrm{N}=60)$ & 0.782 & 0.764 & 0.666 \\
Group 1 $(\mathrm{N}=124)$ & 0.867 & 0.782 & 0.808 \\
Group 2 $(\mathrm{N}=100)$ & 0.852 & 0.742 & 0.805 \\
\hline
\end{tabular}

V-RQOL - Voice Related Quality of Life questionnaire.

SE - Social-Emotional domain, PF - Physical Functioning domain. Other abbreviations as in Table 1.

The results in the study group and control group differ significantly (in $t$ test: $\mathrm{p}<0.001$ ). Moreover, the V-RQOL standard total scores in the non-randomized group 2 are worse: $\mathrm{p}<0.001$ than in the control group, with mean values of 83.99 , and similarly the results are worse for the Social-Emotional domain (mean $(\mathrm{M})=84.13)$ and Physical Functioning domain $(\mathrm{M}=83.76)$.

\section{V-RQOL cut-off point}

The Receiver Operating Characteristic (ROC) curve with AUC was constructed for diagnostic evaluation of the V-RQOL test. The best cut-off that maximizes the

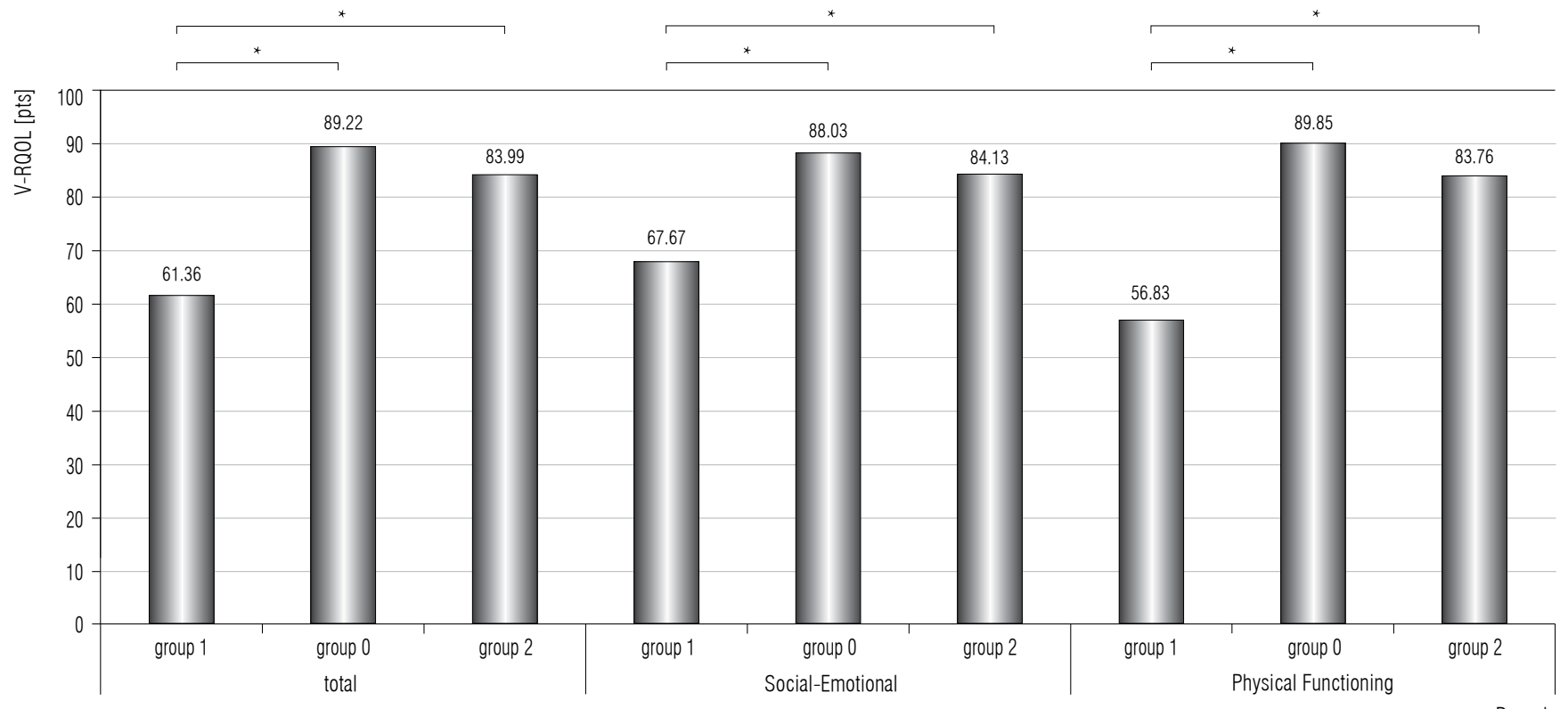

Group 0 - control group of normophonic subjects, group 1 - professional voice users with voice disorders, group 2 - non-randomized group of professional voice users with and without voice. ${ }^{*} \mathrm{p}<0.001$.

Fig. 3. Comparison of the Voice Related Quality of Life (V-RQOL) scores in studied groups of problems showing standard results of V-RQOL - total score, Social-Emotional and Physical Functioning scale 


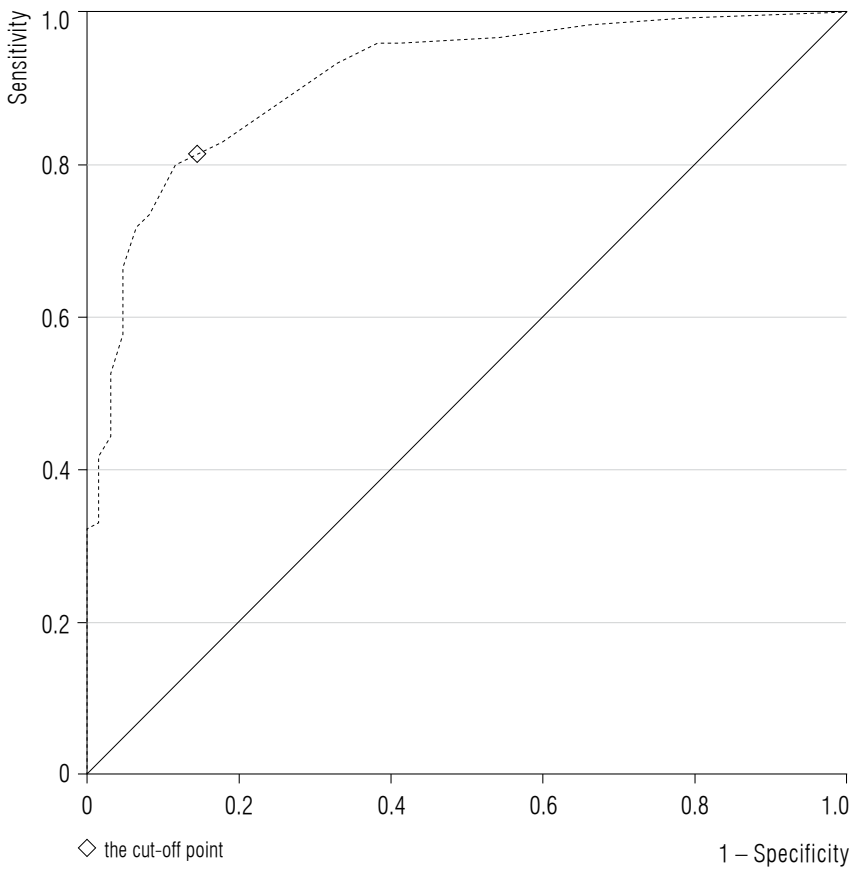

AUC (area under curve) $=0.910, \mathrm{p}<0.001$.

Fig. 4. Receiver Operating Characteristic (ROC) curve for the Voice Related Quality of Life (V-RQOL) test calculated on the basis of V-RQOL standard total scores in group 0 (control group of normophonic subjects) and group 1 (professional voice users with voice disorders

screening value of $\mathrm{V}$-RQOL giving the best relationship between sensitivity and specificity was set at the value of 79 . This point, with sensitivity of 0.831 and specificity of 0.183 , shows the test's best ability to discriminate dysphonic and normophonic subjects (Figure 4).

The analysis of the determined AUC (Table 3) has shown that the accuracy of the V-RQOL as a diagnostic test is excellent: AUC $=0.91, \mathrm{p}<0.001$. The Figure 5 shows ROC curves for the particular items of the V-RQOL questionnaire. From among the 10 vari-

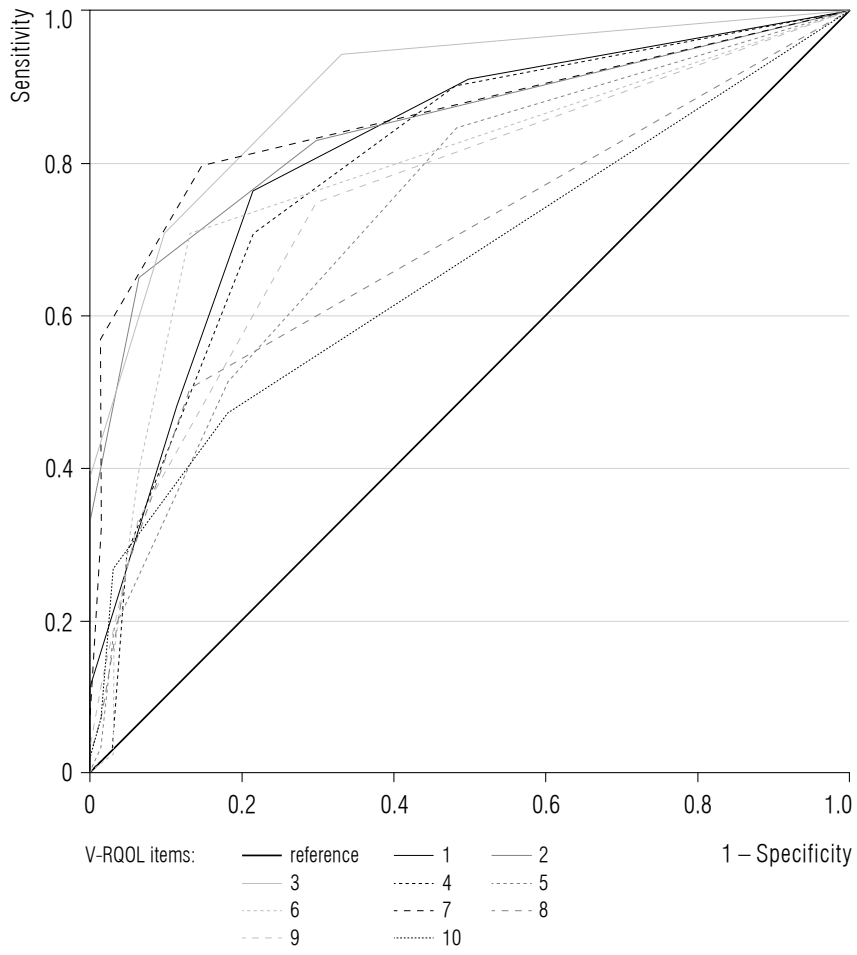

Voice Related Quality of Life (V-RQOL) items: 1 - "I have trouble speaking loudly or being heard in noisy situations," 2 - "I run out of air and need to take frequent breaths when speaking," 3 - "I sometimes do not know what will come out when I begin to speak," 4 - "I am sometimes getting depressed (because of my voice)," 5 - "I am sometimes anxious and frustrated (because of my voice)," 6 - "I have trouble doing my job or practicing my profession," 7 - "I have trouble using the telephone," 8 - "I avoid going out socially," 9 - "I have to repeat myself to be understood," 10 - "I have become less outgoing."

Fig. 5. Sensitivity and specificity of the V-RQOL scale's items calculated on the basis of V-RQOL standard total scores in group 0 (control group of normophonic subjects) and group 1 (professional voice users with voice disorders)

ables of the V-RQOL scale (Table 3), the best discriminating power (the highest value of AUC) is observed for item 3 (the statement: "I sometimes do not know what will come out when I begin speaking"): AUC $=0.895$,

Table 3. Area under the curve (AUC) for the V-RQOL scale's items calculated on the basis of V-RQOL standard total scores in group 0 (control group of normophonic subjects) and group 1 (professional voice users with voice disorders)

\begin{tabular}{|c|c|c|c|c|c|c|c|c|c|c|c|}
\hline Variable & \multicolumn{11}{|c|}{ V-RQOL items $s^{* *}$} \\
\hline AUC & 0.910 & 0.895 & 0.858 & 0.845 & 0.811 & 0.792 & 0.786 & 0.750 & 0.735 & 0.690 & 0.660 \\
\hline $\mathrm{p}^{*}$ & $<0.001$ & $<0.001$ & $<0.001$ & $<0.001$ & $<0.001$ & $<0.001$ & $<0.001$ & $<0.001$ & $<0.001$ & $<0.001$ & $<0.001$ \\
\hline
\end{tabular}

p - probability in the Receiver Operating Characteristic (ROC) curve significance test.

* AUC is significant (area under the ROC curve $>0.5$ ).

** The items are listed according to the highest AUC determined: 3 - "I sometimes do not know what will come out when I begin to speak," 7 - "I have trouble using the telephone," 2 - "I run out of air and need to take frequent breaths when speaking," 1 - "I have trouble speaking loudly or being heard in noisy situations,"

4 - "I am sometimes getting depressed (because of my voice)," 6 - "I have trouble doing my job or practicing my profession," 9 - "I have to repeat myself to be understood,"

5 - "I am sometimes anxious and frustrated (because of my voice)," 8 - "I avoid going out socially," 10 - "I have become less outgoing."

Other abbreviations as in Table 2. 
$\mathrm{p}<0.001$, followed by item 7 ("I have trouble doing my job or practicing my profession because of my voice"): AUC $=0.835, \mathrm{p}<0.001$, and 2 ("I run out of air and need to take frequent breaths when talking"): $\mathrm{AUC}=0.845$, $\mathrm{p}<0.001$. The area under the curve is significantly higher than 0.7 for 8 out of 10 variables of the scale, which indicates that the questions correctly differentiate between normophonic and dysphonic subjects [21].

\section{V-RQOL in diagnose-based subgroups of the study group}

Voice problems reported in the V-RQOL by patients in the study group 1 were confirmed by objective voice evaluation: the acoustic analysis of voice, maximum phonation time (MPT) measurement and VLS. The results showed that the mean values exceeded the threshold of the norm for all the examined parameters: Jitter $=0.72(\mathrm{SD}=1.54), \mathrm{RAP}=0.48(\mathrm{SD}=1.38)$, $\mathrm{PPQ}=0.46(\mathrm{SD}=1.08)$, Shimmer $=6.6(\mathrm{SD}=6.8)$, $\mathrm{APQ}=5.17(\mathrm{SD}=4.66), \mathrm{NHR}=3.16(\mathrm{SD}=1.88)$. The mean values of MPT were also considerably reduced: $12.32 \mathrm{~s}(\mathrm{SD}=6.16)$, which indicated diminished efficiency of vocal tract.

The performed otolaryngological examination with VLS allowed to diagnose 3 distinct voice disorder subgroups: vocal nodules, glottal insufficiency, and hyperfunctional dysphonia without benign vocal fold lesions. The Table 4 presents the V-RQOL results (total and sub-scales) for the particular voice disorder sub-groups. In all sub-groups the mean result of the V-RQOL questionnaire was lower than the cut-off point of 79 determined in the course of this study.

Table 4. Comparison of the V-RQOL scores for the total study group 1 (occupational voice users with voice disorders confirmed by VLS) and the sub-groups of dysphonic subjects established on the basis of VLS diagnosis

\begin{tabular}{|c|c|c|c|}
\hline \multirow{2}{*}{ Study group } & \multicolumn{3}{|c|}{ V-RQOL } \\
\hline & total $^{*}$ & $\mathrm{SE}^{* *}$ & $\mathrm{PF}^{* * *}$ \\
\hline Group $1($ total $)(\mathrm{N}=124)$ & 61.22 & 67.34 & 56.80 \\
\hline $\begin{array}{l}\text { persons with hyperfunctional } \\
\text { dysphonia }(\mathrm{N}=33)\end{array}$ & 59.72 & 65.56 & 55.53 \\
\hline $\begin{array}{l}\text { persons with glottal insufficiency } \\
(\mathrm{N}=29)\end{array}$ & 59.28 & 65.00 & 57.07 \\
\hline persons with vocal nodules $(\mathrm{N}=28)$ & 64.96 & 71.79 & 60.04 \\
\hline others $(\mathrm{N}=34)$ & 62.26 & 68.68 & 57.74 \\
\hline
\end{tabular}

VLS - videolaryngostroboscopy.

${ }^{*} \mathrm{p}=0.597,{ }^{* *} \mathrm{p}=0.539,{ }^{* * *} \mathrm{p}=0.753$.

Other abbreviations as in Table 1 and 2 .
The comparison of the total V-RQOL scores shows a minimal discrepancy between the voice-related quality of life results reported by patients with hyperfunctional dysphonia and those with glottal insufficiency (59.72 and 59.28, respectively) in comparison to those with vocal nodules (64.96). The results are better among patients with vocal nodules than patients with hyperor hyperfunction of glottis, in the total scores as well as in PF and SE domains (Table 4).

\section{DISCUSSION}

Currently, complex assessment of voice, including VLS, the perceptual and acoustic analysis does not include vital information about the effects the voice dysfunction leave on an individual's quality of life [5]. It is important for the diagnosis process to understand the perspective of a patient who experiences voice problems. Measuring what the subject perceives about voice condition is essential, particularly for occupational voice users. This study has looked at the screening utility of the V-RQOL measure for diagnosing occupational voice disorders.

Our research proved a high correlation between the 2 instruments: a commonly used VHI and a shorter, easier to use in clinical practice tool - the V-RQOL. The coefficient of determination describing linear regression between these tests for all the study subjects $(\mathrm{N}=284)$ reached the value: $\mathrm{R}^{2}=0.7266$, which was confirmed by Pearson's coefficient: -0.873 . The data is consistent with the results of similar studies conducted in the past $[22,23]$ and also previously demonstrated in our pilot study on the subject [24].

Additionally, in our current study we have found the satisfactory correlation between both tests in the group of subjects with objectively confirmed (by means of the VLS and acoustic analysis) voice disorders (group 1): Pearson's correlation coefficient $-0.821, \mathrm{R}^{2}=0.6729$. Since both questionnaires correlate high on a significant level, it may be assumed that the V-RQOL with 10 questions produces similar results to the longer 30-item VHI and may thus replace it to facilitate the screening process.

In addition to assessing the applicability of the V-RQOL in the self-assessment of voice disorders we measured the internal consistency of the Polish version of the questionnaire. Cronbach's $\alpha$ for the examined study group in our studies was 0.867 . This result, confirming high reliability of the test is in accordance with other studies: 0.89 [20], 0.91 [12], and 0.92 [22].

The next objective of this research was to study the clinical utility of the V-RQOL for screening occupational 
voice disorders. Therefore the relationship between sensitivity and specificity was examined by means of the ROC curve. The cut-off point at the V-RQOL, distinguishing the subjects with and without voice disorders, was equal to 79. The analysis of the determined area under the curve AUC has shown that the accuracy of V-RQOL as a diagnostic test is excellent: $\mathrm{AUC}=0.91, \mathrm{p}<0.001$.

Behlau et al. [21], researching the efficiency and cutoff values of self-assessment instruments on the impact of voice disorders, underline that the AUC indicates the accuracy of the voice diagnostic test if the areas reach the value $>0.7$. It should be pointed out that in our research, out of 10 items comprising the V-RQOL, in the case of 8 of them the AUC value is above 0.7. This attests to the correct selection of questions reflecting the most typical vocal problems reported by occupational voice users. The literature data regarding the cut-off point of the V-RQOL questionnaire is scarce up to date, however, in the research conducted in Brazil on a vast etiologically non-homogeneous dysphonic group, it was determined at 91.25 [21].

Looking at the discrepancy of 12.5 pts between the above mentioned result and this obtained in our study, the much lower cut-off point established in our research may be justified by 2 factors. Firstly, our study was conducted on a homogenous group - occupational voice users, and secondly, the subjects' voice disorders were objectively confirmed by a thorough phoniatric examination with the VLS and acoustic analysis.

In our study, the mean standard V-RQOL score in the study group with diagnosed occupational voice disorders was 61.8. This result is higher than in the original study conducted by Hogikyan and Sethuraman (the standard total score $(\mathrm{M})=53.5)$ [20], but considerably lower in comparison to other studies - for instance by Aaby and Heimdal (the standard total score $(M)=74.5)$ [12], Sielska-Badurek et al. (the standard total score $(M)=75.5)$ [22] or Gasparini and Beklau (the standard total score $(\mathrm{M})=84.2)$ [15].

Our result may be related to the subject sample, as all the dysphonic individuals in the study group were occupational voice users with recognized voice pathologies. Moreover, people who rely on their voice for work tend to pay greater attention to its quality and are more likely to see any vocal symptoms as a deterioration in the overall quality of life whereas non-professional voice users may perceive lower voice quality as a secondary problem.

It should be stressed that the study did not indicate significant discrepancy between voice-related quality of life among subjects with diverse occupation-related voice pathologies. In the study the following subgroups of patients were diagnosed by means of the VLS - vocal nodules, hypofunctional dysphonia with incomplete glottal closure and hyperfunctional dysphonia without benign vocal fold lesions.

The mean V-RQOL score among subjects with vocal nodules (64.96) was better (higher) than among subjects with hyperfunctional dysphonia (59.72) and glottal insufficiency (59.28), which indicates that nodules (organic dysphonia) do not cause greater impact on quality of life among professional users than functional dysphonia. It is obvious that vocal nodules could have detrimental effect on quality of voice (e.g., in the case of singers they may cause loss of the higher frequencies), which is important for vocal performers [25]. However, they tend to have a lesser impact on vocal efficiency [26] which is important in spoken voice professions e.g., teachers, clerks, telemarketers, and the representatives of the above mentioned professional groups in particular have been examined in the study.

This observation is of great importance given that in many European Union countries and all over the world only vocal nodules are recognized as occupation-related voice disease having detrimental effect on the health, which gives basis for certification of voice disability [2]. It has been underlined in literature data that the impact of professional voice disorders is undervalued as occupational safety and health $(\mathrm{OSH})$ issue $[1,27,28]$. The study has confirmed this statement and demonstrated that functional voice disorders may also lead to diminished biopsychosocial work conditions in the case of voice professionals.

Future research should be conducted on a larger population and should investigate the voice-related quality of life of occupational voice users within particular vocally demanding professions.

\section{CONCLUSIONS}

The current study indicates that the V-RQOL test may be useful to distinguish subjects with voice disorders. It may be concluded that the score of 79 should be considered as the cut-off point in the Polish version of the V-RQOL questionnaire to discriminate between individuals with and without voice disorders. This tool may prove useful in screening procedures for occupational voice disorders and therefore may be used as a primary source of referral in clinical practice, particularly in occupational medicine. 
This simple and short self-assessment tool may be helpful for the clinician in the early diagnostic process for a better identification of vocal problem of individuals, including occupational voice users. This is the first study which gives grounds for application of the V-RQOL scale as a simple screening tool for professional voice users.

\section{REFERENCES}

1. De Jong F. An introduction to the teacher's voice in a biopsychosocial perspective. Folia Phoniatr Logop. 2010;62: 5-8, https://doi.org/10.1159/000239058.

2. Calcinoni O, Niebudek-Bogusz E. Occupational voice. In: Rubin J, Sataloff R, Korovin G, editors. Diagnosis and treatment of voice disorders. 4th ed. San Diego: Plural Publishing; 2014. p. 735-62.

3. Bermudez de Alvear RM, Martinez GA, Baron FJ, Hernandez-Mendo A. An interdisciplinary approach to teachers' voice disorders and psychosocial working conditions. Folia Phoniatr Logop. 2010;62:24-34, https://doi.org/10.1159/ 000239060.

4. Leeper WR, Fung K, Beaudin PG, Doyle PC. Voice-related quality of life in patients with benign vocal fold lesions. J Otolaryngol. 2008;37(3):423-9.

5. Moradi N, Pourshahbaz A, Soltani M, Javadipour S. Cutoff point at Voice Handicap Index used to screen voice disorders among Persian speakers. J Voice. 2013;27(1):130.1-5, https://doi.org/10.1016/j.jvoice.2012.08.007.

6. Aboras Y, El-Banna M, El-Magraby R, Ibrahim A. The relationship between subjective self-rating and objective voice assessment measures. Logoped Phoniatr Vocol. 2010;35(1):34-8, https://doi.org/10.3109/14015430903 582128.

7. Niebudek-Bogusz E, Śliwińska-Kowalska M. An overview of occupational voice disorders in Poland. Int J Occup Med Environ Health. 2013;26(5):659-69, https://doi. org/10.2478/s13382-013-0146-7.

8. Merrill RM, Roy N, Lowe J. Voice-related symptoms and their effects on quality of life. Ann Otol Rhinol Laryngol. 2013;122(6):404-11, https://doi.org/10.1177/00034894 1312200610 .

9. Williams NR. Occupational groups at risk of voice disorders: A review of the literature. Occup Med (Lond). 2003;53(7):456-60, https://doi.org/10.1093/occ $\mathrm{med} / \mathrm{kqg} 113$.

10. Carding P. Occupational voice disorders: Is there a firm case for industrial injuries disablement benefit? Logoped Phoniatr Vocol. 2007;32(1):47-8, https://doi.org/10.1080/ 14015430600881901.
11. World Health Organization. The World Health Organization Quality of Life instruments. Measuring the quality of life. Geneva: The Organization; 1997. p. 1-15.

12. Aaby C, Heimdal JH. The Voice-Related Quality of Life (V-RQOL) measure - A study on validity and reliability of the Norwegian version. J Voice. 2013;27(2):258.29-33, https://doi.org/10.1016/j.jvoice.2012.10.007.

13. Tutya AS, Zambon F, Oliveira G, Behlau M. Comparison of V-RQOL, VHI and VAPP scores in teachers. Rev Sov Bras Fonoaudiol. 2011;16:273-81, https://doi.org/10.1590/ S1516-80342011000300007.

14. Romak JJ, Orbelo DM, Maragos NE, Ekbom DC. Correlation of the Voice Handicap Index-10 (VHI-10) and Voice-Related Quality of Life (V-RQOL) in patients with dysphonia. J Voice. 2014;28(2):237-40, https://doi.org/ 10.1016/j.jvoice.2013.07.015

15. Gasparini G, Behlau M. Quality of life: Validation of the Brazilian Version of the Voice-Related Quality of Life (V-RQOL) measure. J Voice. 2009;23(1):76-81, https:// doi.org/10.1016/j.jvoice.2007.04.005.

16. Jones SM, Carding PN, Drinnan MJ. Exploring the relationship between severity of dysphonia and voice-related quality of life. Clin Otolaryngol. 2006;31(5):411-7, https:// doi.org/10.1111/j.1749-4486.2006.01291.x.

17. Murry T, Medrado R, Hogikyan ND, Aviv JE. The relationship between ratings of voice quality and quality of life measures. J Voice. 2004;18(2):183-92, https://doi.org/10.10 16/j.jvoice.2003.11.003.

18. Niebudek-Bogusz E, Kuzańska A, Woznicka E, Sliwinska-Kowalska M. Assessment of the Voice Handicap Index as a screening tool in dysphonic patients. Folia Phoniatr Logop. 2011;63(5):269-72, https://doi.org/10.1159/ 000324214.

19. Wiskirska-Woźnica B, Wojnowski W. [The smokers voice self assessment based on Voice Handicap Index (VHI)]. Przegl Lek. 2009;66(10):565-6. Polish.

20. Hogikyan ND, Sethuraman G. Validation of an instrument to measure voice-related quality of life (V-RQOL). J Voice. 1999;13(4):557-69, https://doi.org/10.1016/S08921997(99)80010-1.

21. Behlau M, Madazio G, Moreti F, Oliveira G, Dos Santos Lde M, Paulinelli BR, et al. Efficiency and cutoff values of self-assessment instruments on the impact of a voice problem. J Voice. 2016;30(4):506.9-18, https://doi. org/10.1016/j.jvoice.2015.05.022.

22. Sielska-Badurek E, Rzepakowska A, Sobol M, OsuchWójcikiewicz E, Niemczyk K. Adaptation and validation of the Voice-Related Quality of Life measure into Polish. J Voice. 2016;30(6):773.7-12, https://doi.org/10.1016/j.jvoice.2015.11.014. 
23. Portone CR, Hapner ER, McGregor L, Otto K, Johns MM, 3rd. Correlation of the Voice Handicap Index (VHI) and the Voice-Related Quality of Life Measure (V-RQOL). J Voice. 2007 Nov;21(6):723-7, https://doi.org/10.1016/j.jvoice.20 06.06.001.

24. Morawska J, Niebudek-Bogusz E, Zaborowski K, Wiktorowicz J, Śliwińska-Kowalska M. V-RQOL as a tool for assessing the impact of voice disorders on the quality of life in occupational voice users. Otorynolaryngologia 2015;14(2):96-103.

25. Rubin JS, Yanagisawa E. Benign vocal fold pathology through the eyes of the laryngologist. In: Rubin JS, Sataloff RT, Korovin GS, editors. Diagnosis and treatment of voice disorders. San Diego: Plural Publishing; 2014. p. 95-115.
26. Van Stan JH, Mehta DD, Zeitels SM, Burns JA, Barbu AM, Hillman RE. Average ambulatory measures of sound pressure level, fundamental frequency, and vocal dose do not differ between adult females with phonotraumatic lesions and matched control subjects. Ann Otol Rhinol Laryngol. 2015;124(11):864-74, https://doi. org/10.1177/0003489415589363.

27. Rossi-Barbosa LA, Barbosa MR, Morais RM, de Sousa KF, Silveira KF, Gama AC, et al. Self-reported acute and chronic voice disorders in teachers. J Voice. 2016;30(6): 755.25-33, https://doi.org/10.1016/j.jvoice.2015.08.003.

28. Martins RH, Pereira ER, Hidalgo CB, Tavares EL. Voice disorders in teachers. A review. J Voice. 2014;28(6):716-24, https://doi.org/10.1016/j.jvoice.2014.02.008.

This work is available in Open Access model and licensed under a Creative Commons Attribution-NonCommercial 3.0 Poland License - http://creativecommons.org/licenses/by-nc/3.0/pl/deed.en. 